\title{
Spin flipping with an rf dipole and a full Siberian snake
}

\author{
B. B. Blinov, Ya. S. Derbenev, T. Kageya, D. Yu. Kantsyrev,* A. D. Krisch, V. S. Morozov,* \\ D. W. Sivers, ${ }^{\dagger}$ and V. K. Wong \\ Randall Laboratory of Physics, University of Michigan, Ann Arbor, Michigan 48109-1120 \\ V.A. Anferov, P. Schwandt, and B. von Przewoski \\ Indiana University Cyclotron Facility, Bloomington, Indiana 47408-0768
}

(Received 5 June 2000; published 25 October 2000)

\begin{abstract}
We recently used an $\mathrm{rf}$ dipole magnet to study the spin flipping of a $120 \mathrm{MeV}$ horizontally polarized proton beam stored in the presence of a nearly full Siberian snake in the Indiana University Cyclotron Facility Cooler Ring. We flipped the spin by ramping the rf dipole's frequency through an rf-induced depolarizing resonance. After optimizing the frequency ramp parameters, we used multiple spin flips to measure a spin-flip efficiency of $86.5 \pm 0.5 \%$. The spin-flip efficiency was apparently limited by the field strength in the rf dipole. This result indicates that spin flipping a stored polarized proton beam should be possible in high energy rings such as the Brookhaven Relativistic Heavy Ion Collider and HERA where Siberian snakes are certainly needed and only dipole rf-flipper magnets are practical.
\end{abstract}

PACS numbers: 29.27.Bd, 29.27.Hj, 41.75.Ak

Polarized beam experiments are now a major component of the programs in storage rings such as the Indiana University Cyclotron Facility (IUCF) Cooler Ring [1], the MIT-Bates Storage Ring [2], the Brookhaven Relativistic Heavy Ion Collider [3], and HERA at DESY [4]. Frequent reversals of the beam polarization direction can significantly reduce the systematic errors in an experiment's spin asymmetry measurements. An rf solenoid was used earlier to spin flip a horizontally polarized proton beam stored in the Cooler Ring containing a Siberian snake [5] with $97 \pm 1 \%$ spin-flip efficiency [6]. However, a solenoid's spin rotation decreases linearly with energy because of the Lorentz contraction of its $\int B d l$; thus, a solenoid is impractical for spin flipping in high energy rings. Fortunately, a dipole's spin rotation is energy independent. Therefore, we recently used an rf dipole to spin flip a $120 \mathrm{MeV}$ horizontally polarized proton beam stored in the IUCF Cooler Ring with a nearly full Siberian snake.

In any flat circular accelerator or storage ring with no horizontal magnetic fields, each proton's spin precesses around the vertical fields of the ring's dipole magnets. The spin tune $\nu_{s}$, which is the number of spin precessions during one turn around the ring, is proportional to the proton's energy

$$
\nu_{s}=G \gamma,
$$

where $G=(g-2) / 2=1.792847$ is the proton's gyromagnetic anomaly and $\gamma$ is its Lorentz energy factor.

This vertical spin precession can be perturbed by the horizontal rf magnetic field from either an rf solenoid or an rf dipole. This perturbation can induce an rf depolarizing

\footnotetext{
*Also at Moscow State University, Moscow, Russia.

${ }^{\dagger}$ Also at Portland Physics Institute, Portland, OR 97201.
}

resonance, which can be used to flip the spin direction of the ring's stored polarized protons [6,7]. The frequency $f_{r}$, at which an rf-induced depolarizing resonance occurs, is given by

$$
f_{r}=f_{c}\left(k \pm \nu_{s}\right)
$$

where $f_{c}$ is the proton's circulation frequency and $k$ is an integer. Sweeping the rf magnet's frequency through $f_{r}$ can flip the spin. The Froissart-Stora equation [8] relates the beam's polarization after crossing the resonance $P_{f}$ to its initial polarization $P_{i}$,

$$
P_{f}=P_{i}\left\{2 \exp \left[\frac{-\left(\pi \epsilon f_{c}\right)^{2}}{\Delta f / \Delta t}\right]-1\right\},
$$

where $\epsilon$ is the resonance strength and $\Delta f / \Delta t$ is the resonance crossing rate, while $\Delta f$ is the frequency range during the ramp time $\Delta t$.

The apparatus used for this experiment, including the rf dipole, the IUCF Cooler Ring, and the polarimeter, were discussed earlier [6,9-23] and are shown in Fig. 1. The $120 \mathrm{MeV}$ horizontally polarized proton beam in the Cooler Ring was obtained using the new cooler injector polarized ion source (CIPIOS) [24] and the new cooler injection synchrotron (CIS) [25]. The beam polarization after the $7 \mathrm{MeV}$ linac was $59 \pm 2 \%$. At $120 \mathrm{MeV}$, the circulation frequency in the Cooler Ring was $f_{c}=1.59784 \mathrm{MHz}$.

With a nearly full Siberian snake in the ring, the spin tune $\nu_{s}$ is very near but not exactly equal to $1 / 2$. Therefore, at $120 \mathrm{MeV}$, Eq. (2) implies that two closely spaced rf depolarizing resonances should be centered around

$$
1.5 f_{c}=2.39676 \mathrm{MHz}
$$

with their frequencies at

$$
\begin{aligned}
& f_{r}^{-}=f_{c}\left(2-\nu_{s}\right), \\
& f_{r}^{+}=f_{c}\left(1+\nu_{s}\right) .
\end{aligned}
$$




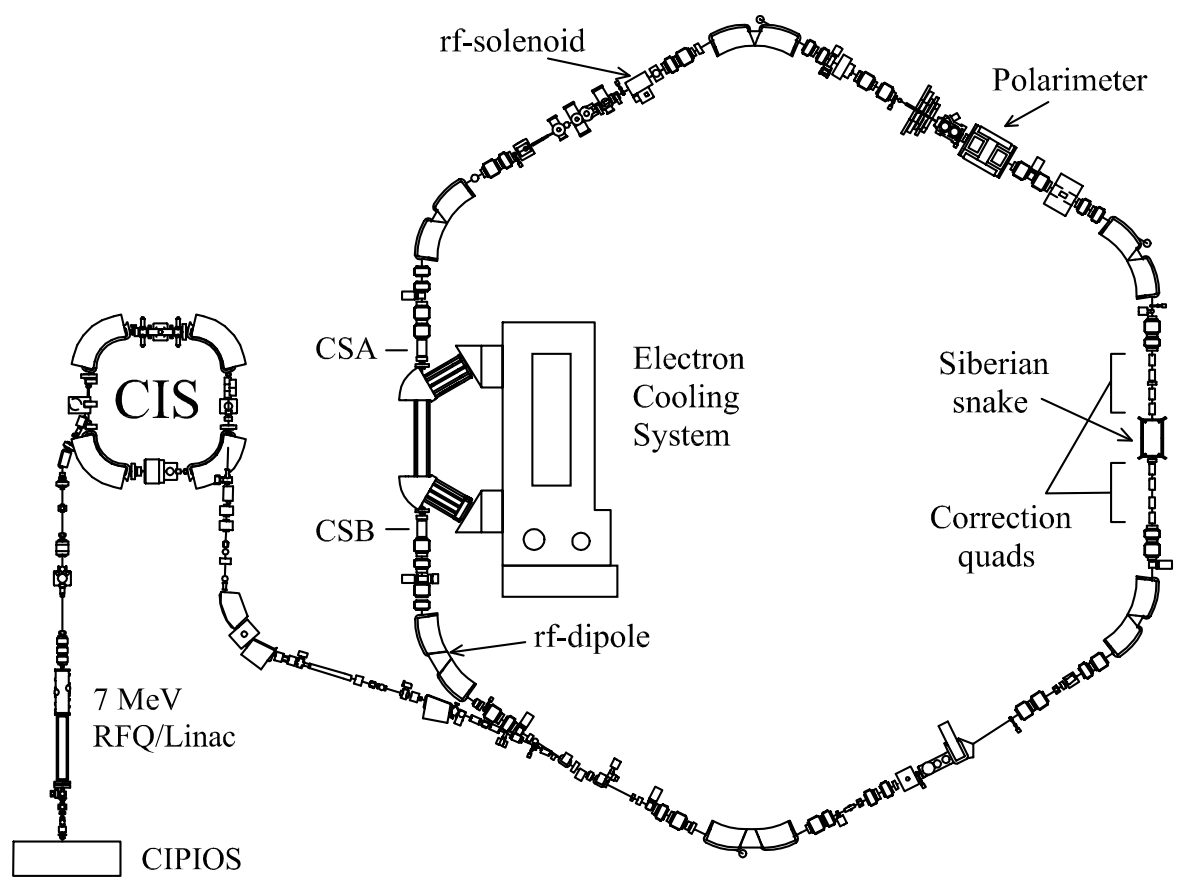

FIG. 1. Layout of the IUCF Cooler Ring with its new CIS injector and CIPIOS polarized ion source. Also shown in the Cooler Ring are the rf dipole, the rf solenoid, the polarimeter, and the Siberian snake.

Since our snake strength $s$ was about $1.01, \nu_{s}$ was about 0.505 ; thus, the $f_{r}^{-}$resonance should have a frequency slightly below $1.5 f_{c}$.

We first determined the $f_{r}^{-}$resonance's frequency to be near $2.384 \mathrm{MHz}$ by using the much stronger rf solenoid. Then we used a simple $L C$ resonant circuit to increase the rf dipole's input voltage to $71 \mathrm{~V}$ rms corresponding to an $\int B d l$ amplitude of $0.06 \mathrm{~T} \mathrm{~mm} \mathrm{rms.} \mathrm{We}$ then measured, at different dipole frequencies, the radial polarization, which is about $89 \%$ of the total horizontal polarization at the position of our polarimeter at $120 \mathrm{MeV}$. This measured radial polarization is plotted against the $\mathrm{rf}$ dipole's frequency in Fig. 2. The curve is a second-order Lorentzian fit to the data with a resonance frequency of $f_{r}^{-}=2384040 \pm 90 \mathrm{~Hz}$ and a width $w$ of $970 \pm 20 \mathrm{~Hz}$.

The separation $\delta$ of this $f_{r}^{-}$resonance from the central frequency of $1.5 f_{c}$ allows one to determine the spin tune $\nu_{s}$, and thus the Cooler Ring's total snake strength $1+\Delta s$, using the approximation $[13,26]$

$$
\begin{aligned}
\Delta s & =2\left(\nu_{s}-0.5\right)=2 \delta / f_{c} \\
& =\frac{2(2.39676-2.38404)}{1.59784}=0.8 \% .
\end{aligned}
$$

To study spin flipping, we crossed this rf-induced resonance by linearly ramping the rf-dipole's frequency, through the measured $f_{r}^{-}$, from $f_{r}^{-}-5$ to $f_{r}^{-}+5 \mathrm{kHz}$, with various ramp times $\Delta t$, while measuring the beam polarization after each frequency ramp. The measured radial polarization is plotted against the ramp time in Fig. 3. Note that, after a very rapid change, the polarization's magnitude seems constant for ramp times above
$200 \mathrm{msec}$. We fit this measured polarization to a modified [6] Froissart-Stora formula

$$
P_{f}=P_{i}\left\{(1+\eta) \exp \left[\frac{-\left(\pi \epsilon f_{c}\right)^{2}}{\Delta f / \Delta t}\right]-\eta\right\},
$$

where $\eta$ is the spin-flip efficiency; from this fit, we obtained an $87 \pm 2 \%$ spin-flip efficiency.

We tried to further increase the spin-flip efficiency by varying the rf dipole's frequency range $\Delta f$ with its ramp time $\Delta t$ set at $500 \mathrm{msec}$, its amplitude set at $0.06 \mathrm{~T} \mathrm{~mm}$,

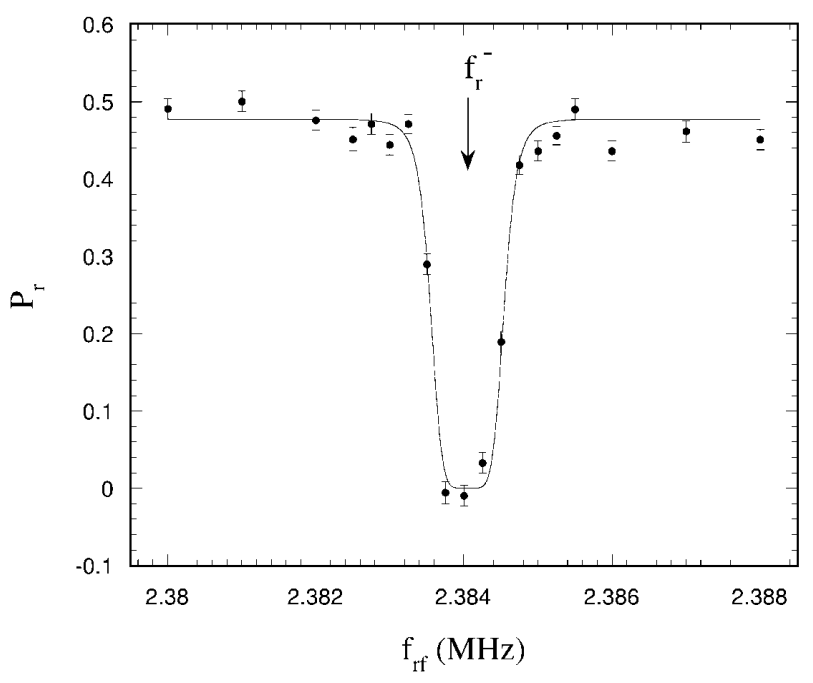

FIG. 2. The measured radial proton polarization at $120 \mathrm{MeV}$ is plotted against the $\mathrm{rf}$ dipole's frequency. The $\mathrm{rf}$ dipole's $\int B d l$ was $0.06 \mathrm{~T} \mathrm{~mm}$. The curve is a fit using a second-order Lorentzian. The arrow shows $f_{r}^{-}$. 


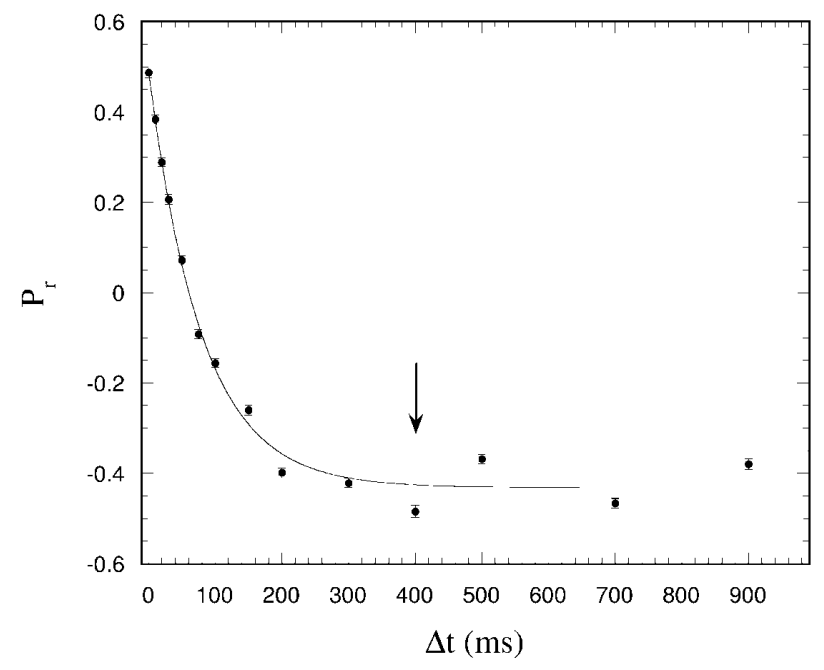

FIG. 3. The measured radial proton polarization at $120 \mathrm{MeV}$ is plotted against the rf dipole ramp time $\Delta t$. The frequency range $\Delta f$ was $10 \mathrm{kHz}$, and the rf dipole's $\int B d l$ was $0.06 \mathrm{~T} \mathrm{~mm}$. The curve is a fit to the data using Eq. (7). The arrow shows the $\Delta t$ value used for Fig. 4.

and its range centered around $2.38404 \mathrm{MHz}$. The data indicated that the spin-flip efficiency had a broad maximum near $\Delta f=10 \mathrm{kHz}$.

After setting $\Delta t$ and $\Delta f$ to maximize the spin-flip efficiency, we more precisely determined this efficiency by measuring the radial polarization after many spin flips. We varied the number of spin flips while keeping, for each spin flip, the ramp time, the frequency range, and the rf voltage all fixed. This radial polarization is plotted against the number of spin flips in Fig. 4. We fit this data using

$$
P_{n}=P_{i} \cdot \eta^{n}
$$

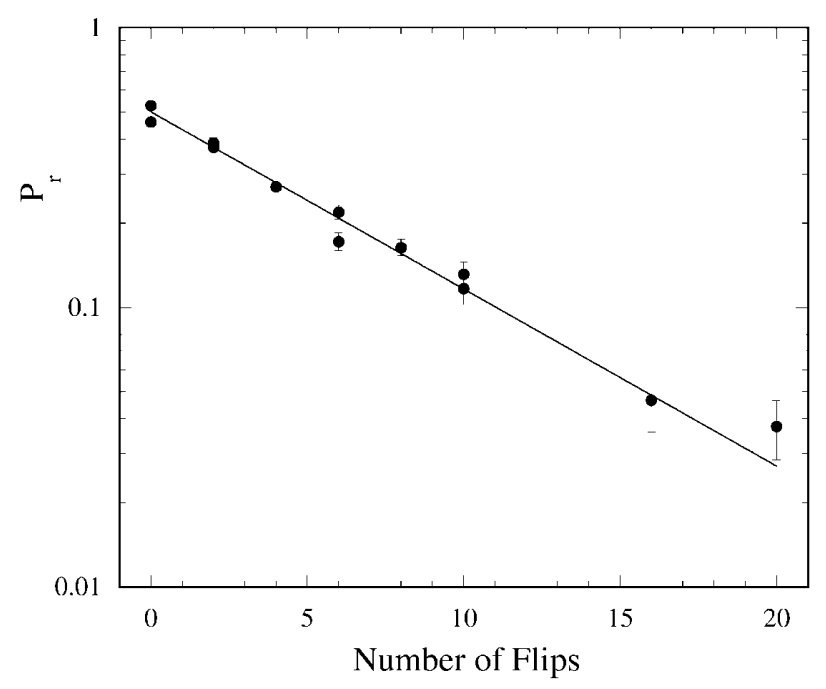

FIG. 4. The measured radial proton polarization at $120 \mathrm{MeV}$ is plotted against the number of spin flips. The rf dipole's frequency ramp time $\Delta t$ was $400 \mathrm{msec}$, its frequency range $\Delta f$ was $10 \mathrm{kHz}$, and its $\int B d l$ was $0.06 \mathrm{~T} \mathrm{~mm}$. The curve is a fit to the data using Eq. (8). where $P_{n}$ is the measured radial beam polarization after $n$ spin flips, $P_{i}$ is the initial polarization, $\eta$ is the spin-flip efficiency, and $n$ is the number of spin flips. The best fit gave a spin-flip efficiency of $86.4 \pm 0.5 \%$. The spin-flip efficiency was apparently limited by the strength of the rf dipole's field; we hope to further increase the spin-flip efficiency by further increasing the rf-dipole's $\int B d l$.

In summary, we used an rf dipole to spin flip a stored $120 \mathrm{MeV}$ horizontally polarized proton beam with a nearly full Siberian snake in the IUCF Cooler Ring. Combining the data from Figs. 3 and 4, the measured spin-flip efficiency is $86.5 \pm 0.5 \%$. This result indicates that spin flipping a stored polarized proton beam should be possible in $100 \mathrm{GeV}$ to $1 \mathrm{TeV}$ rings where Siberian snakes are certainly needed and only dipole rf-flipper magnets are practical.

We would like to thank J. M. Cameron, A. S. Belov, V. P. Derenchuk, G.W. East, D. L. Friesel, T. Rinckel, W. T. Sloan, E. J. Stephenson, and the entire Indiana University Cyclotron Facility staff for the successful operation of the Cooler Ring with its new CIS and CIPIOS. We are grateful to R. Baiod, A. W. Chao, E. D. Courant, F. Z. Khiari, S. Y. Lee, A. M. T. Lin, W. Lorenzon, P. S. Martin, H. O. Meyer, M. G. Minty, C. Ohmori, R. A. Phelps, R.E. Pollock, L. G. Ratner (deceased), T. Roser, A.D. Russell, H. Sato, T. Toyama, and B. Yankama for their help with earlier parts of this experiment. This research was supported by grants from the U.S. Department of Energy and the U.S. National Science Foundation.

[1] H. O. Meyer et al., Phys. Rev. Lett. 81, 3096 (1998); B. von Przewoski et al., Phys. Rev. C 58, 1897 (1998).

[2] R. Alarcon et al., BLAST Collaboration, 1999 Bates Report, No. 2-39, 1999.

[3] Y. Makdisi, in High Energy Spin Physics: 11th International Symposium, AIP Conf. Proc. No. 343 (AIP, New York, 1995), p. 75.

[4] SPIN Collaboration, University of Michigan Reports No. UM-HE 96-20, 1996, and No. UM-HE 99-05, 1999.

[5] Ya. S. Derbenev and A. M. Kondratenko, Sov. Phys. Dokl. 20, 562 (1976).

[6] B. B. Blinov et al., Phys. Rev. Lett. 81, 2906 (1998); V. A. Anferov et al., in Proceedings of the 13th International Symposium on High Energy Spin Physics, edited by N.E. Tyurin et al. (World Scientific, Singapore, 1999), p. 503.

[7] B. W. Montague, Phys. Rep. 113, 35 (1984).

[8] M. Froissart and R. Stora, Nucl. Instrum. Methods 7, 297 (1960).

[9] D. A. Crandell et al., Phys. Rev. Lett. 77, 1763 (1996).

[10] V. A. Anferov et al., Phys. Rev. ST Accel. Beams 3, 041001 (2000).

[11] A. D. Krisch et al., Phys. Rev. Lett. 63, 1137 (1989).

[12] J. E. Goodwin et al., Phys. Rev. Lett. 64, 2779 (1990).

[13] M. G. Minty et al., Phys. Rev. D 44, R1361 (1991).

[14] V. A. Anferov et al., Phys. Rev. A 46, R7383 (1992).

[15] R. Baiod et al., Phys. Rev. Lett. 70, 2557 (1993).

[16] R. A. Phelps et al., Phys. Rev. Lett. 72, 1479 (1994). 
[17] B. B. Blinov et al., Phys. Rev. Lett. 73, 1621 (1994).

[18] D. D. Caussyn et al., Phys. Rev. Lett. 73, 2857 (1994).

[19] C. Ohmori et al., Phys. Rev. Lett. 75, 1931 (1995).

[20] L. V. Alexeeva et al., Phys. Rev. Lett. 76, 2714 (1996).

[21] R. A. Phelps et al., Phys. Rev. Lett. 78, 2772 (1997).

[22] C. M. Chu et al., Phys. Rev. E 58, 4973 (1998).

[23] B. B. Blinov et al., Phys. Rev. ST Accel. Beams 2, 064001 (1999).

[24] V.P. Derenchuk and A. S. Belov, in Polarized Gas Targets and Polarized Beams, edited by Roy J. Holt and Michael A. Miller, AIP Conf. Proc. No. 421 (AIP, New York, 1998), p. 422.

[25] D. L. Friesel and S. Y. Lee, in Proceedings of the 1997 Particle Accelerator Conference (PAC97), Vancouver, Canada (IEEE, Piscataway, NJ, 1998), p. 935.

[26] There was also an inadvertent Type-3 snake in the electron cooling section of the Cooler Ring whose strength was typically less than $1 \%$. 